\title{
Detection of simulated incipient furcation involvement by CBCT: an in vitro study using pig mandibles
}

\section{Otavio Shoiti Umetsubo Bruno Felipe Gaia \\ Felipe Ferreira Costa Marcelo Gusmão Paraiso Cavalcanti}

Department of Stomatology, School of Dentistry, University of São Paulo, São Paulo, SP, Brazil.
Declaration of Interests: The authors certify that they have no commercial or associative interest that represents a conflict of interest in connection with the manuscript.

Corresponding Author: Marcelo Gusmão Paraiso Cavalcanti E-mail:mpgcaval@usp.br

Received for publication on Mar 06, 2012 Accepted for publication on Apr 29, 2012

\begin{abstract}
The aim of the present study was to test the reproducibility, sensitivity, and specificity of cone-beam computed tomography (CBCT) in detecting incipient furcation involvement. Fifteen macerated pig mandibles, with intact second molar teeth and preserved adjacent cortical areas, were used. Simulated lesions were created in the furcation region of these teeth by applying $70 \%$ perchloric acid in up to four possible buc$\mathrm{cal} /$ lingual sites in the right/left sides of each mandible. The mandibles were then submitted to a CBCT scan. Two blinded and calibrated experienced oral and maxillofacial radiologists interpreted the exams. Furcation involvement was also assessed in the regions without simulated lesions. CBCT showed high levels of accuracy, ranging from $78 \%$ to $88 \%$. The variations in Kappa values for intra- and inter-observer agreement (0.41-0.59) were considered moderate. CBCT can be considered a reliable and accurate method for detecting incipient furcation involvement.
\end{abstract}

Descriptors: Cone-beam Computed Tomography; Periodontics; Furcation Defects.

\section{Introduction}

Furcation involvement is one of the major problems in the treatment of periodontal disease, and is directly associated with tooth loss. Therefore, furcation lesions must be detected with accuracy at an early stage of the disease. ${ }^{1,2}$ Periapical and interproximal intraoral radiographs are widely accepted as valuable tools for establishing the diagnosis and guiding the treatment of periodontal disease, mainly to assess the pattern and extent of bone resorption. ${ }^{3,4}$ These characteristics can be clearly viewed in the interproximal alveolar bone. However, intraoral radiographs have the inherent inaccuracy of a two-dimensional exam. In the free faces, structural overlapping usually impairs the demarcation of bone loss extension in the vestibular and lingual cortical areas. ${ }^{5}$ Studies have shown a significant advantage of CBCT over intraoral radiographs owing to its three-dimensional capability to assess buccal and lingual surfaces. ${ }^{6,7}$ Recently, cone-beam computed tomography (CBCT) has been used to evaluate dental structures, providing volumetric and 3D information of submillimeter resolution and without any image distortion of the anatomic structures. ${ }^{8-10}$ Several studies have evaluated the potential of this imaging technique both in vivo (in advanced disease conditions, such as chronic periodontitis) $)^{5,11,12}$ and in vitro (using simulated lesions created 
with high-speed spherical drills).6,13-17 These studies have shown high levels of accuracy for CBCT in detecting lesions owing to lesion size (in cases of chronic periodontal disease) or to well-defined lesion boundaries resulting from a methodology that uses drills (in vitro studies).

In previous studies, the performance of periapical radiography in detecting larger periodontal lesions proved inferior to that of CBCT. ${ }^{5-7,11-17}$ In clinical studies, ${ }^{5,11,12}$ the primary purpose of radiographic examination was not as much to perform an early detection of periodontitis, as it was to characterize the disease and support the treatment follow-up.

In vitro studies ${ }^{6,13-17}$ simulate lesions by producing them with high-speed spherical drills. This methodology makes it possible to create defects with defined contours ${ }^{18}$ and to simulate an advanced stage of the disease.

The aim of the present study was thus to test the reproducibility, sensitivity, and specificity of conebeam computed tomography (CBCT) in detecting incipient furcation involvement.

\section{Methodology}

\section{Selection of specimens}

The present study was submitted to the Ethics and Research Committee of our institution for approval under protocol no. 104/2010.

Fifteen mandibles of young adult pigs (previously slaughtered for human consumption) were used. The mandibular second molars were intact and the adjacent cortex was preserved. ${ }^{19}$ The mandibles were boiled in water (for 8 hours, twice) and their soft tissues (gingivae, mucosa, and muscles) were removed. The mandibles were then washed with neutral soap to remove lipid remnants. Before the simulated lesions were created, the specimens were assessed clinically and tomographically in order to assess whether they had any original bone defect that could be misinterpreted in the ensuing diagnostic imaging. Any specimen that had a bone defect was excluded from the study.

\section{Simulating lesions in the alveolar process in the furcation region}

Simulated lesions were created in the furca- tion region of the second molars in up to four possible sites (buccally and lingually, and in the right and left sides) of each mandible, for a total of 60 possible sites. These sites were divided into a control group (with no lesion) and a test group (with a lesion), which were then analyzed simultaneously. Twenty of these sites were selected randomly, so that a mandible could have a variable number $(0-4)$ of lesions in the furcation region of the molar teeth, bilaterally. One of the authors, whose job was not to evaluate the images (O.S.U.), created the lesions. The lesions were made to simulate incipient bone loss caused by periodontal disease at an early stage. Two-millimeter cotton pledgets soaked in $70 \%$ perchloric acid were kept in contact with the bone for 2 hours (Figure 1); after each application, the mandibles were washed for 1 min under tap water, according to the methodology used in previous studies. ${ }^{19,20}$

\section{Image acquisition}

CBCT images of the mandibles were acquired with a single apparatus (i-CAT ${ }^{\circledR}$ New Generation, Imaging Sciences International, Hatfield, USA), using the following acquisition protocol:

- voxel size: $0.2 \mathrm{~mm}$,

- acquisition time: $26.9 \mathrm{~s}$,

- tube voltage: $120 \mathrm{kV}$,

- filament current: $5 \mathrm{~mA}$,

- field of view (FOV): $6 \times 16 \mathrm{~cm}$.

The images were obtained with the axial plane oriented along and leveled with the mandible base, since this is a position similar to that occurring in a clinical setting. The analysis was conducted independently, and on separate occasions, by two oral and maxillofacial radiologists (F.F.C. and B.F.G.) with extensive experience in interpreting CBCT scans, using Xoran software (v. 3.1.62, Xoran Technologies Inc., Ann Arbor, USA). The initial assessments of Observer 1 and Observer 2 were called Observation 1 and Observation 2, respectively. Standardization included training in the tomographic features involved in detecting furcation lesions. For this purpose, CBCT images of other specimens, with and without lesions, were presented to the observers. They were asked to assess the second 
mandibular molar region and identify any possible furcation involvement, even in the regions where no simulated lesion was created. They were blinded to any information on the procedures performed and on the conditions of the teeth. Each observer analyzed the images independently using multiplanar reconstructed images (axial, coronal and sagittal, simultaneously), in an independent workstation (Figure 2). They were free to use the software visualization tools (contrast, magnification, and window width and level). When interpreting the images, the room lights were turned off.

The interpretation time was not restricted, and the second assessment of the same images was repeated within a 14-day interval by the same observers to produce Observation 1' and Observation 2'. The absence or presence of a bone defect was assessed in each of the four sites on the 15 mandibles $(\mathrm{n}=60)$ to compare the imaging diagnosis of the lesions with that of the gold standard (macerated mandible).

Statistical analysis was carried out using the validity and Kappa tests (SPSS software, v. 17.0.0, SPSS Inc., Chicago, USA). The values for the Kappa (к) coefficient were calculated to assess the intraand inter-observer agreement, which were classified according to the following criteria:

- low $(\kappa=0.20-0.39)$,

- moderate $(\kappa=0.40-0.59)$, and

- substantial $(\kappa=0.60-0.79)$.

The values for sensitivity and specificity were estimated in order to check the agreement of the assessments against the gold standard, and to distinguish the false positives and false negatives observed in each group.
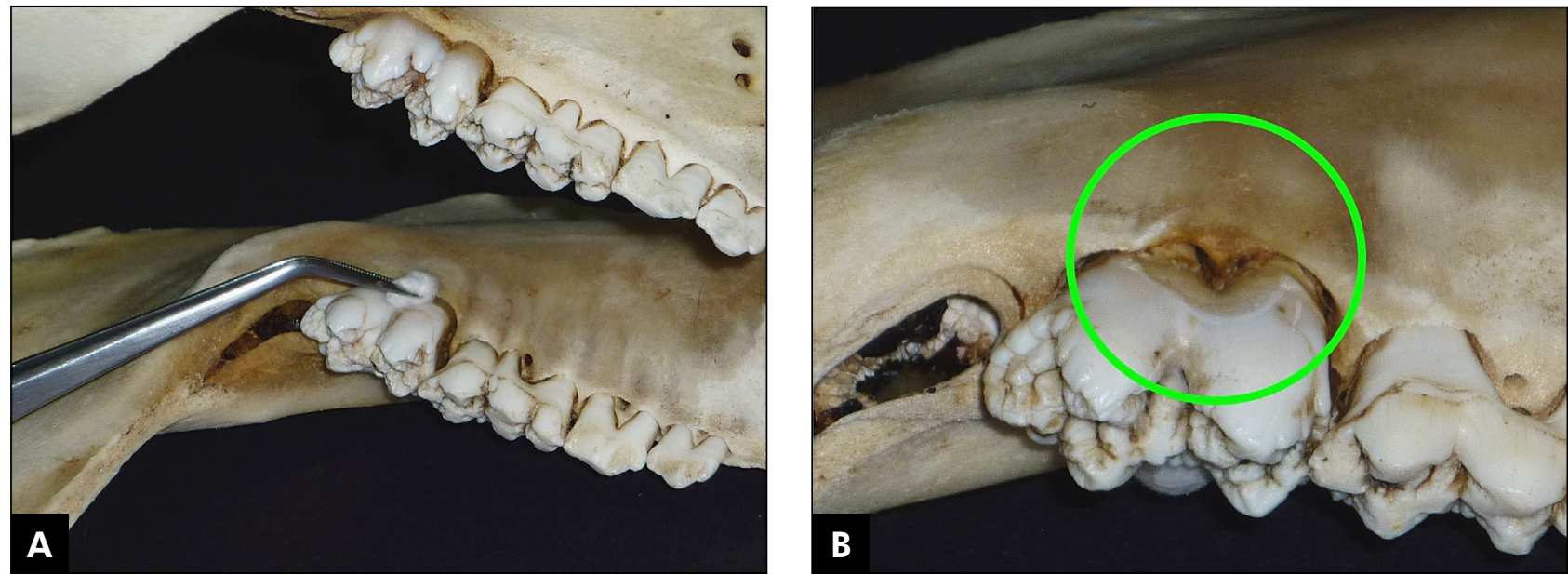

Figure 1 - Positioning of the cotton fragment (A), and chemical lesion simulation. (B) Final aspect, two hours after acid application.
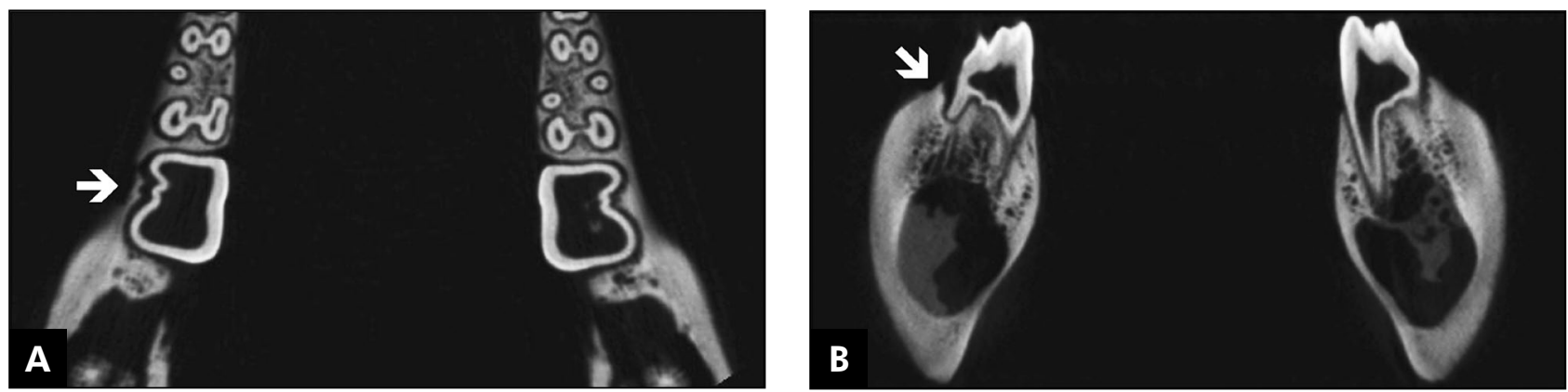

Figure 2 - CBCT axial (A) and coronal (B) images. The lesion on the right vestibular surface (arrows) was detected by both observers. 


\section{Results}

The kappa values for the intra-observer agreement in detecting incipient furcation involvement ranged from 0.41 to 0.55 . The kappa values for inter-observer agreement, ranged from 0.52 to 0.59 . All of these values were considered as representing moderate levels of agreement (Table 1).

Table 2 shows the results for overall sensitivity, specificity, and accuracy in detecting incipient furcation involvement. These data represent all observations for both observers. CBCT showed moderate sensitivity (ranging from $50 \%$ to $75 \%$ ), represented by the true positive results of detecting incipient furcation involvement. However, high specificity (ranging from $93 \%$ to $100 \%$ ) was observed, represented by the correlation between the true negative results and the sites where there was actually no lesion. The accuracy of CBCT (ranging from $78 \%$ to $88 \%$ ) was represented by the proportion between true positive and true negative results, considered simultaneously in relation to all results

\section{Discussion}

According to $\mathrm{Mol},{ }^{4}$ an imaging examination (either intraoral radiography or CBCT) in periodontology will benefit the patient only if it can influence the treatment. Therefore, an exam should be indicated only if the additional information it provides will make a difference in patient management and if this difference will have an impact on the outcomes that are important to him/her. The same author emphasizes that three-dimensional (3D) information may have advantages over information obtained from two-dimensional (2D) examinations. However, it is necessary to evaluate whether an increase in diagnostic efficacy has an impact on both the treatment and the overall results for the patient. Corbet et al. ${ }^{3}$ reported that neither helical nor multislice CT offer any favorable cost-benefit ratio or therapeutic advantage to periodontal practice. These authors disagree with $\mathrm{Mol}^{4}$ and state that CBCT should be used more often because it involves a lower radiation dose. We agree with the statements made by $\mathrm{Mol}^{4}{ }^{4}$ Vandenberghe et al. ${ }^{16}$ and Walter et al. ${ }^{21}$ that a diagnostic method must produce a clinical outcome, and we emphasize that the biological effects of ionizing
Table 1 - Intra- and inter-observer agreement.

\begin{tabular}{l|l|l}
\hline & Values & Agreement \\
\hline Intra-observer 1 & 0.55 & Moderate \\
\hline Intra-observer 2 & 0.41 & Moderate \\
\hline Inter-observer 1 $\times 2$ & 0.59 & Moderate \\
\hline Inter-observer 1' $\times 2^{\prime}$ & 0.52 & Moderate \\
\hline
\end{tabular}

Table 2 - Sensitivity, specificity and accuracy values for both observers in both analyses.

\begin{tabular}{c|c|c|c|c}
\hline \multicolumn{2}{|c|}{} & Sensitivity & Specificity & Accuracy \\
\hline \multirow{2}{*}{$\begin{array}{c}\text { First } \\
\text { assessment }\end{array}$} & Observation 1 & $70 \%$ & $98 \%$ & $88 \%$ \\
\cline { 2 - 5 } & Observation 2 & $75 \%$ & $90 \%$ & $85 \%$ \\
\hline $\begin{array}{c}\text { Second } \\
\text { assessment }\end{array}$ & Observation 1' $^{\prime}$ & $50 \%$ & $100 \%$ & $83 \%$ \\
\cline { 2 - 5 } & Observation 2' & $50 \%$ & $93 \%$ & $78 \%$ \\
\hline
\end{tabular}

radiation must always be taken into account, even though the radiation dose from CBCT is reduced.

Naitoh et al. ${ }^{5}$ presented a case of upper molars with pocket depth measurements of $4 \mathrm{~mm}$ (mesiobuccal), $6 \mathrm{~mm}$ (disto-buccal), $6 \mathrm{~mm}$ (mesio-palatal), and $5 \mathrm{~mm}$ (disto-palatal). They also used 3D reconstructed images from CBCT to characterize alveolar bone loss. However, this procedure is controversial, since several factors involved in 3D image acquisition may interfere with the results, namely:

- the operator's subjective analysis,

- the acquisition parameters $(\mathrm{kV}, \mathrm{mA}$, voxel size and FOV size), and

- the software parameters (threshold, reconstruction algorithms). ${ }^{22}$

Walter et al. ${ }^{11,12}$ presented clinical cases with severe chronic periodontitis, in which a furcation involvement was clinically detected. CBCT was used for lesion assessment and treatment planning, not for the early detection and prevention of lesions. Grimard et al. ${ }^{8}$ evaluated the outcomes of a regenerative treatment using intraoral radiographs and CBCT images. According to Naitoh et al. ${ }^{5}$ and Walter et al., ${ }^{11,12}$ the subjects included in these studies presented advanced stages of periodontitis. In the present study, our goal was to analyze CBCT images of incipient furcation lesions using a rigorous 
experimental control. Lesions of this size were not analyzed in previous studies.

Misch et al., ${ }^{6}$ Grimard et al. ${ }^{8}$ and Noujeim et al. ${ }^{15}$ addressed periodontal lesions on the proximal surfaces of teeth. Interproximal lesions render CBCT a less decisive tool for the prognosis of affected teeth (access to and hygiene of a tooth's mesial and distal faces are easier than access/hygiene in the furcation region). Periodontal resorption begins with immunological and inflammatory events, leading to bone demineralization. Therefore, it is possible that bone loss is not related to the degree of inflammation or the qualitative nature of the inflammation, but rather to where the inflammation is located in the bone and to how long it persists.

In the in vitro studies of Misch et al., ${ }^{6}$ Mengel et al., ${ }^{13}$ Vandenberghe et al. ${ }^{16,17}$ and Noujeim et al., ${ }^{15}$ drills were used to simulate lesions. In these studies, CBCT images demonstrated good reliability in both detecting the lesions and measuring their dimensions. However, lesions simulated with a drill would represent a more advanced stage of the disease, which could be detected by either periodontal probing or intraoral radiography. Fuhrmann et al. ${ }^{23}$ also used drills to simulate lesions, and reported that helical CT is a reliable technique to classify furcation involvement both vertically and horizontally.

In previous studies, Mol \& Balasundaram ${ }^{14}$ concluded that $\mathrm{CBCT}$ is better than intraoral radiography in terms of both aiding in establishing the diagnosis and providing quantitative information on periodontal bone levels. However, the authors admitted that CBCT failed to provide sufficient spatial resolution in cases where the diagnostic decisions depended on small details. Periodontal resorption starts with inflammatory and immune events, resulting in bone demineralization. At an initial stage, the cavities are small and have irregular borders. ${ }^{24,25}$ We believe that lesions are more difficult to detect with either intraoral radiography or CBCT at this stage. In the simulations shown in the present study, we intended to explore the extent to which additional information obtained by CBCT images can impact the periodontal treatment. We simulated the bone loss caused by inflammation occurring more deeply, or otherwise not easily detected clinically.
We also simulated a situation in which CBCT could detect a bone resorption process in time to allow removing the cause of inflammation and resuming the physiological cycle, thus allowing bone formation and restoration of the lost bone.

The lesions created in the present study were smaller than those produced in previous studies, ${ }^{6,13-17}$ with the aim of simulating an initial stage of bone resorption. Studies validating the use of CBCT to detect lesions, simulating an early stage of periodontitis, with a rigorous experimental control, were not found in the related literature.

The research published by Mol \& Balasundaram $^{14}$ demonstrated low levels of intra-observer agreement. In our study, moderate levels of intraand inter-observer agreement were obtained. The intra-observer agreement may have increased owing to the advanced technology of the equipment. A high amount of false-negative diagnoses were reached, which may be explained by both the reduced lesion dimensions and the changes in the technique used to simulate the lesions.

It is clear that CBCT is still not the first choice for periodontal bone support imaging. This function continues to be well performed by periapical radiographs. However, CBCT can help in cases where periapical radiographs are not sufficiently illustrative owing to overlapping structures. Nevertheless, CBCT imaging can be indicated for other applications, such as assessments for implant placement and periodontal furcation surgery. Even though incipient furcation lesions are not considered major indications for CBCT, any changes detected in the furcation region of molar teeth in patients scanned for other reasons should nevertheless be reported.

Following the guidelines of imaging interpretation criteria, the whole volume of data obtained by a CBCT examination should be carefully assessed, regardless of the original diagnostic question underlying the examination.

\section{Conclusion}

CBCT imaging showed high accuracy and moderate reproducibility in the detection of incipient furcation involvement. Based on these results, we believe that this technique can be considered a re- 
liable tool for detecting incipient furcation involvement, thus expanding the applicability reported by other authors, who tested the CBCT technique as applied to larger furcation lesions, which were not created chemically using acid.

\section{Acknowledgements}

We wish to acknowledge the National Council for Scientific and Technological Development (CNPq), Brasília, DF, Brazil, for financially supporting the work of Dr. Marcelo Cavalcanti, Uni-

\section{References}

1. Hishikawa T, Izumi M, Naitoh M, Furukawa M, Yoshinari $\mathrm{N}$, Kawase H, et al. The effect of horizontal X-ray beam angulation on the detection of furcation defects of mandibular first molars in intraoral radiography. Dentomaxillofac Radiol. 2010 Feb;39(2):85-90.

2. Walter C, Weiger R, Zitzmann NU. Periodontal surgery in furcation-involved maxillary molars revisited-an introduction of guidelines for comprehensive treatment. Clin Oral Investig. $2011 \mathrm{Feb} ; 15(1): 9-20$.

3. Corbet EF, Ho DKL, Lai SML. Radiographs in periodontal disease diagnosis and management. Aust Dent J. 2009 Sep;54 Suppl 1:S27-43.

4. Mol A. Imaging methods in periodontology. Periodontol 2000. 2004 Feb;34(1):34-48.

5. Naitoh M, Yamada S, Noguchi T, Ariji E, Nagao J, Mori K, et al. Three-dimensional display with quantitative analysis in alveolar bone resorption using cone-beam computerized tomography for dental use: a preliminary study. Int J Periodontics Restorative Dent. 2006 Dec;26(6):607-12.

6. Misch KA, Yi ES, Sarment DP. Accuracy of cone beam computed tomography for periodontal defect measurements. J Periodontol. 2006 Jul;77(7):1261-6.

7. De Faria Vasconcelos K, Evangelista K, Rodrigues C, Estrela C, de Sousa T, Silva M. Detection of periodontal bone loss using cone beam CT and intraoral radiography. Dentomaxillofac Radiol. 2012 Jan;41(1):64-9.

8. Grimard BA, Hoidal MJ, Mills MP, Mellonig JT, Nummikoski $\mathrm{PV}$, Mealey BL. Comparison of clinical, periapical radiograph, and cone-beam volume tomography measurement techniques for assessing bone level changes following regenerative periodontal therapy. J Periodontol. 2009 Jan;80(1):48-55.

9. Tetradis S, Anstey P, Graff-Radford S. Cone beam computed tomography in the diagnosis of dental disease. J Calif Dent Assoc. 2010 Jan;38(1):27-32.

10. Cavalcanti MG. Cone beam computed tomographic imaging: perspective, challenges, and the impact of near-trend future applications. J Craniofac Surg. 2012 Jan;23(1):279-82. versal Research Project, grant no. 472895/2009-5, and Research Productivity Scholarship, grant no. 303847/2009-3. We also wish to thank the Coordination for the Improvement of Higher Education Personnel (CAPES), Brasília, DF, Brazil, for providing $\mathrm{PhD}$ scholarships to Felipe Costa and Bruno Gaia, and a Master's degree scholarship to Otavio Umetsubo. Additionally, we would like to thank the Kavo Research Institute for providing the CBCT equipment support.

11. Walter C, Kaner D, Berndt DC, Weiger R, Zitzmann NU. Three-dimensional imaging as a pre-operative tool in decision making for furcation surgery. J Clin Periodontol. 2009 Mar;36(3):250-7.

12. Walter C, Weiger R, Zitzmann NU. Accuracy of three-dimensional imaging in assessing maxillary furcation involvement. J Clin Periodontol. 2010 May;37(5):436-41.

13. Mengel R, Candir M, Shiratori K, Flores-de-Jacoby L. Digital volume tomography in the diagnosis of periodontal defects: an in vitro study on native pig and human mandibles. J Periodontol. 2005 May;76(5):665-73.

14. Mol A, Balasundaram A. In vitro cone beam computed tomography imaging of periodontal bone. Dentomaxillofac Radiol. 2008 Sep;37(6):319-24.

15. Noujeim M, Prihoda T, Langlais R, Nummikoski P. Evaluation of high-resolution cone beam computed tomography in the detection of simulated interradicular bone lesions. Dentomaxillofac Radiol. 2009 Mar;38(3):156-62.

16. Vandenberghe B, Jacobs R, Yang J. Diagnostic validity (or acuity) of 2D CCD versus 3D CBCT - images for assessing periodontal breakdown. Oral Surg Oral Med Oral Pathol Oral Radiol Endod. 2007 Sep;104(3):395-401.

17. Vandenberghe B, Jacobs R, Yang J. Detection of periodontal bone loss using digital intraoral and cone beam computed tomography images: an in vitro assessment of bony and/or infrabony defects. Dentomaxillofac Radiol. 2008 Jul;37(5):25260.

18. Tirrell BC, Miles DA, Brown CE, Legan JJ. Interpretation of chemically created lesions using direct digital imaging. J Endod. 1996 Feb;22(2):74-8.

19. Stravopoulos A, Wenzel A. Accuracy of cone beam dental CT, intraoral digital and conventional film radiography for the detection of periapical lesions. An ex vivo study in pig jaws. Clin Oral Investig. 2007 Mar;11(1):101-6.

20. Sogur E, Baksi BG, Gröndahl H-G, Lomcali G, Sen BH. Detectability of chemically induced periapical lesions by limited cone beam computed tomography, intra-oral digital and con- 
ventional film radiography. Dentomaxillofac Radiol. 2009 Oct;38(7):458-64.

21. Walter C, Weiger R, Dietrich T, Lang NP, Zitzmann NU. Does three-dimensional imaging offer a financial benefit for treating maxillary molars with furcation involvement? - A pilot clinical case series. Clin Oral Implants Res. 2012 Mar;23(3):351-8.

22. Hassan B, Couto Souza P, Jacobs R, de Azambuja Berti S, van der Stelt P. Influence of scanning and reconstruction parameters on quality of three-dimensional surface models of the dental arches from cone beam computed tomography. Clin Oral Investig. 2010 Jun;14(3):303-10.
23. Fuhrmann RAW, Bücker A, Diedrich PR. Furcation involvement: comparison of dental radiographs and HR-CT-slices in human specimens. J Periodontal Res. 1997 Jul;32(5):409-18.

24. Graves DT, Li J, Cochran DL. Inflammation and uncoupling as mechanisms of periodontal bone loss. J Dent Res. 2011 Feb;90(2):143-53.

25. Nanci A, Bosshardt DD. Structure of periodontal tissues in health and disease. Periodontol 2000. 2006 Feb;40(1):11-28. 